\title{
Endoscopic mucosal resection for esophageal and gastric mucosal cancers
}

\author{
Haruhiro Inoue MD
}

\begin{abstract}
H Inoue. Endoscopic mucosal resection for esophageal and gastric mucosal cancers. Can J Gastroenterol 1998;12(5):355-359. Accumulated data from surgically resected specimens reveal that mucosal cancers of the esophagus and stomach pose low risk of lymph node metastasis. The author used endoscopic mucosal resection (EMR) as curative treatment in 142 cases of esophageal cancer and 102 cases of stomach cancer. In absolutely indicated cases there has been no local or distant metastasis during the longest period of follow-up (nine years). One perforation and one post-treatment severe stenosis, which was resistant to dilation therapy in the esophagus, were encountered. Deeper layer resection (including partial proper muscle) occurred in the stomach in three cases where the lesions were positioned to the lesser curvature of the upper part of the stomach. Two cases of gastric mucosal resection leaving residual cancer were successfully treated by laser ablation. No case has required further surgery. Resected specimens were contributed to histological evaluation in all cases. In conclusion, EMR can be considered as the first-line treatment for selected cases of early stage esophageal and stomach cancer.
\end{abstract}

Key Words: Endoscopic mucosal resection, Esophageal cancer, Gastric cancer

\section{Résection endoscopique des muqueuses dans les cas de cancer des muqueuses de l'œsophage et de l'estomac}

RÉSUMÉ : Selon les données accumulées à partir de spécimens de résection chirurgicale, les cancers de la muqueuse de l'œsophage et de l'estomac posent un faible risque de métastases des ganglions lymphatiques. Les auteurs ont utilisé la résection endoscopique de la muqueuse comme traitement curatif dans 142 cas de cancers de l'œesophage et dans 102 cas de cancers de l'estomac. Dans les cas d'indication absolue, on n'a noté aucune métastase locale ou distale durant la période de suivi la plus longue (neuf ans). Une perforation et une sténose post-thérapeutique grave qui s'est révélée résistante au traitement de dilatation au niveau de l'œsophage sont survenues. La résection des couches plus profondes (y compris du muscle partiel) a été effectuée au niveau de l'estomac dans trois cas où les lésions étaient situées au niveau de la petite courbure de l'estomac à la partie supérieure de l'estomac. Deux cas de résection de la muqueuse gastrique avec cancer résiduel ont été traités avec succès par ablation au laser. Aucun cas n'a nécessité d'autre chirurgie. Les spécimens réséqués ont été fournis pour évaluation histologique dans tous les cas. En conclusion, la résection endoscopique de la muqueuse peut être envisagée en traitement de première ligne dans certains cas sélectionnés pour le cancer de l'œesophage et de l'estomac aux premiers stades de la maladie.

\section{DIAGNOSIS OF EARLY ESOPHAGEAL CANCER}

Detection of early stage esophageal cancer: Normal esophageal mucosa usually appears at endoscopy as smooth and flat, with a whitish lustrous surface. Early stage esophageal cancer is characterized by changes in colour, lustrelessness and rough surfaces, with marginal step-up or step-down.
However its detection is somewhat difficult in quite minute or early stage lesions because of the lack of the aforementioned typical characteristics. In IIb-type cancerous lesions, no changes can be detected, even with meticulous observation using videoendoscopy.

Fortunately, the iodine-dye staining method is a very use-

First Department of Surgery, Tokyo Medical and Dental University, Tokyo, Japan

Correspondence and reprints: Dr Haruhiro Inoue, Chief of Endoscopic Surgery, First Department of Surgery, Tokyo Medical and Dental University, 1-5-45 Yushima, Bunkyo-Ku, Tokyo 113-8519, Japan. Telephone 81-3-5803-5255, fax 81-3-3817-4126,

e-mail hrio.inoue.srg1@med.tmd.ac.jp 

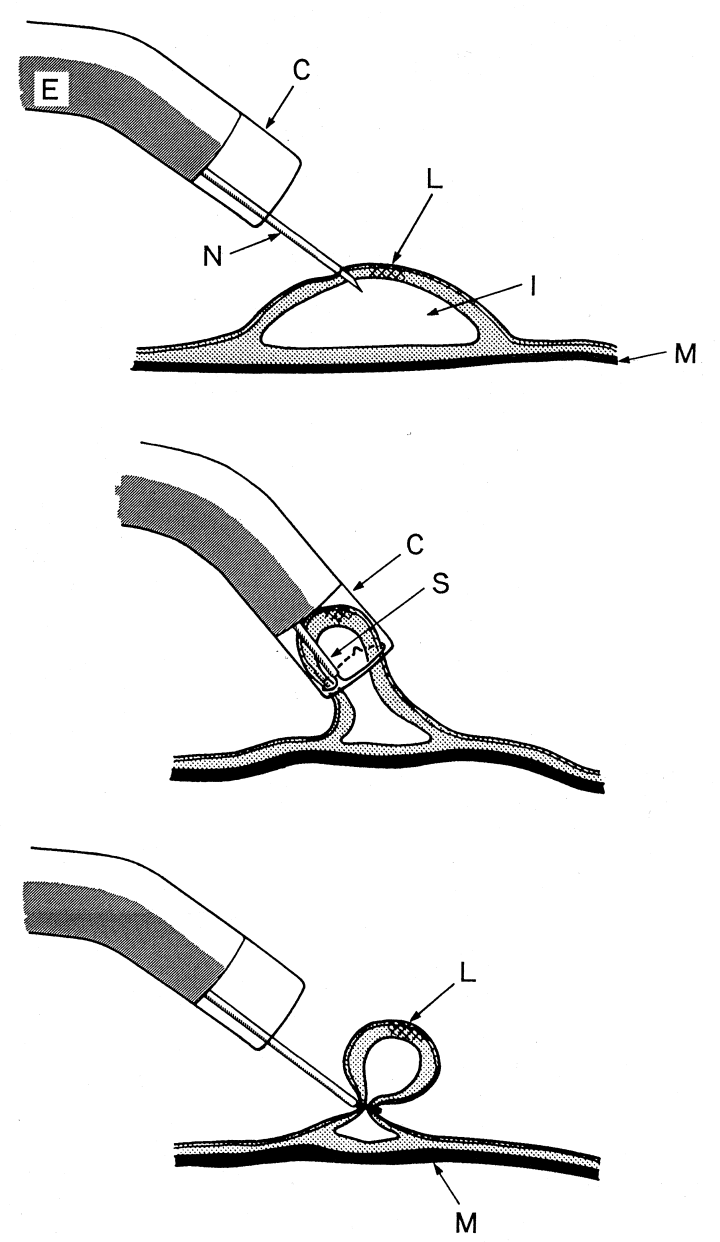

Figure 1) Schema of endoscopic mucosal resection using a cap-fitted endoscope (EMRC). Top Submucosally injected saline (I) lifts mucosa up; Middle After meeting the prelooping condition of the snare $(S)$ wire, target mucosa is sucked inside the EMRC cap (C) attached to the distal end of the endoscope. In prelooping, the opened S wire is fixed along the rim of the cap; Bottom Target mucosa is strangulated by the $S$ wire and resected by electrocautery. L Lesion; M Muscle layer; N Injection needle

ful diagnostic tool in the esophagus. This technique involves spraying iodine dye solution into the esophagus. Normal esophageal epithelium, which normally contains glycogenrich granules, is stained dark brown. Cancerous lesions, which lose glycogen granules in the prickle cell layer, clearly demonstrate an uncoloured area with a clear margin to iodine-stained normal mucosa. Well-demarcated unstained areas under iodine enhancement can be classified as squamous cell carcinoma with high sensitivity and specificity. Endoscopic pinch biopsy is essential for histological confirmation (1).

Indication of mucosal resection: According to data from a Japanese national survey for histological evaluation of surgically resected esophageal cancer specimen (2), mucosal cancer involvement is limited to the proper mucosal layer, affecting only $4 \%$ of the lymph nodes, whereas submucosally invaded cancer has 35\% lymph node metastasis; therefore, patients with mucosal cancer can be considered appropriate candidates for mucosal resection as curative treatment. Technically, even submucosal cancer, if it were lifted by submucosal saline injection, could be resected by this procedure, so submucosal cancer patients may also be candidates for surgery.

\section{DIAGNOSIS OF EARLY STAGE GASTRIC CANCER}

Detection of early stage gastric cancer: The early stage gastric cancer most often encountered is characterized by changes such as reddish colour of the mucosal surface, lustrelessness, rough surface, marginal irregular step-down (type IIc), wide base elevation (type IIa), etc.

Dye-enhanced endoscopy is also useful in detecting cancer of the stomach. Spraying indigocarmine solution enhances the mucosal surface irregularities mentioned above.

Indications of mucosal resection: Absolute indications of mucosectomy in gastric cancer are as follows: differentiated adenocarcinoma; mucosal cancer less than $10 \mathrm{~mm}$ in IIb and IIc lesions without ulcer or ulcer scar; and mucosal cancer less than $20 \mathrm{~mm}$ in IIa lesions.

According to data from the Cancer Institute Hospital in Tokyo (3), which deals with more than 10,000 resected gastric cancer cases, type IIc mucosal cancer less than $10 \mathrm{~mm}$ has no lymph node involvement and type IIc mucosal cancer less than $20 \mathrm{~mm}$ has $0.4 \%$ lymph node involvement. Type IIa mucosal cancer less than $20 \mathrm{~mm}$ has no lymph node metastasis and type IIa mucosal cancer less than $20 \mathrm{~mm}$ has no lymph node involvement; however, less than $30 \mathrm{~mm}$ submucosal cancer has $28.6 \%$ lymph node involvement. Pretherapeutic diagnosis between mucosal and submucosal cancer in type Ila elevated lesion is relatively difficult. In poorly differentiated carcinoma some risks of lymph node metastasis exist even in small lesions; therefore, that type of lesion generally should be excluded from mucosal resection.

\section{TREATMENT OF EARLY STAGE CANCERS BY ENDOSCOPIC MUCOSAL RESECTION}

Introduction: Various local treatments have been used to treat mucosal cancer, such as laser ablation, argon-plasma coagulator and irradiation, but endoscopic mucosal resection (EMR) is the only technique to acquire resected specimens that contribute to histopathological final diagnosis.

Modifying the technique of mucosal resection, we originated the technique of EMR using a cap-fitted endoscope (EMRC), which we believe is the most simple technique to perform mucosectomy anywhere in the gastrointestinal tract.

Principle: The gastrointestinal tract basically consists of two major components: a mucosal layer and a muscle layer. Mucosa grows from internal germ, and muscle is derived from middle germ of viviparity. These two components are attached to each other by loose connective tissue of submucosa and can easily be separated by external force, which is why one can resect just mucosa, leaving the remaining muscle layer intact. 


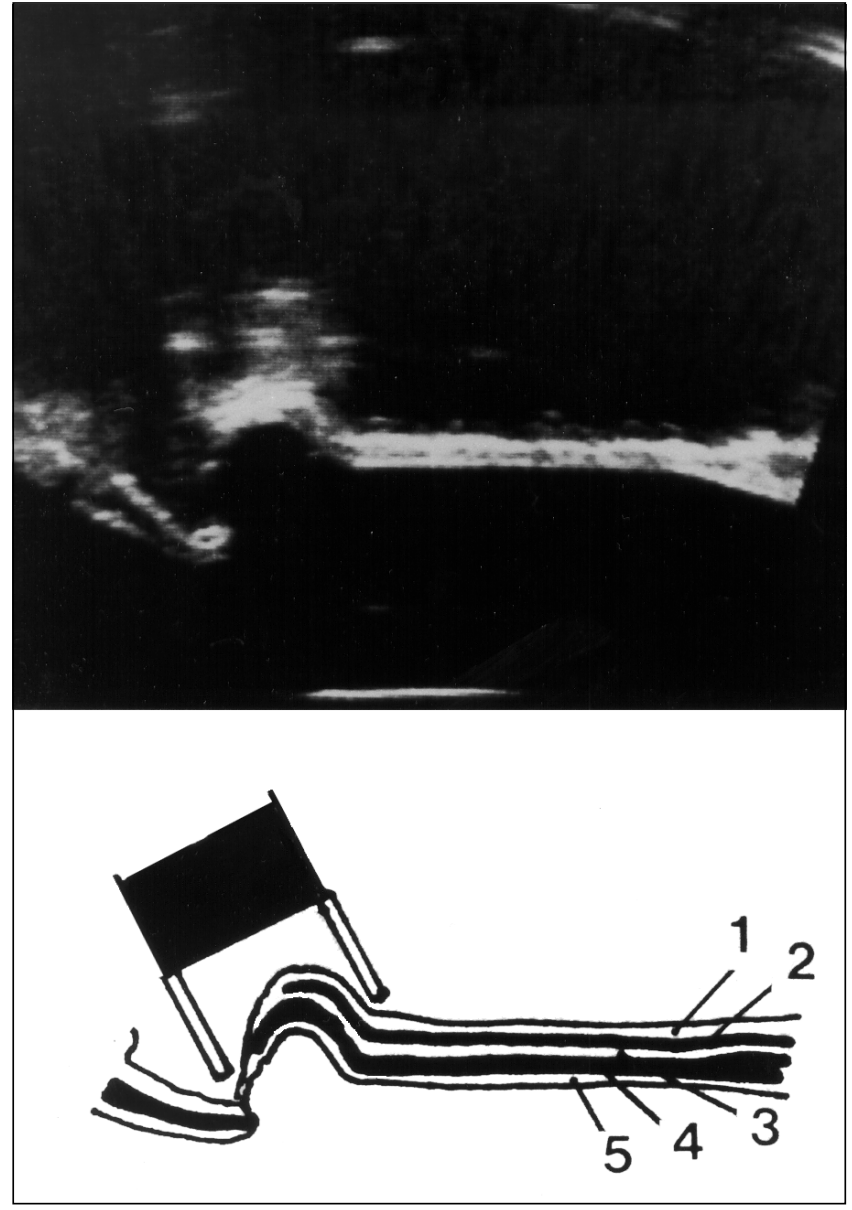

Figure 2) In vitro study of the endoscopic mucosal resection using a capfitted endoscope (EMRC) procedure. All stomach wall is sucked inside the EMRC cap, which entails a risk of strangulating the muscle layer. Numbers indicate the five echo layers

However, because the gastrointestinal wall has only less than $4 \mathrm{~mm}$ of full thickness, special management to avoid the perforation is extremely important. Injection of saline solution into the submucosal layer is the easiest and most effective technique, and avoids major complications during mucosal resection. Lifting of mucosa (in any part of the gastrointestinal tract) is always demonstrated during submucosal saline injection. After sufficient volume of saline injection, the mucosa, including the target lesion, can be safely captured and strangulated by snare wire, and resected by electrocauterization.

History: In 1955, in the era of rigid scope, Rosenberg (4) reported the importance of submucosal saline injection during polypectomy of rectal and sigmoidal polyps. In 1973, Deyhle et al (5) restressed the usefulness of submucosal saline injection to treat sessile colonic polyps by using a flexible endoscope. In 1984 Tada et al (6) advocated mucosal resection for flat and depressed-type mucosal cancer in the stomach.

In 1990 we (7) reported our first experience of mucosal resection in the esophagus by using an originally designed transparent tube. In 1993 we developed the EMRC procedure using a hood-type distal attachment; this technique is con-

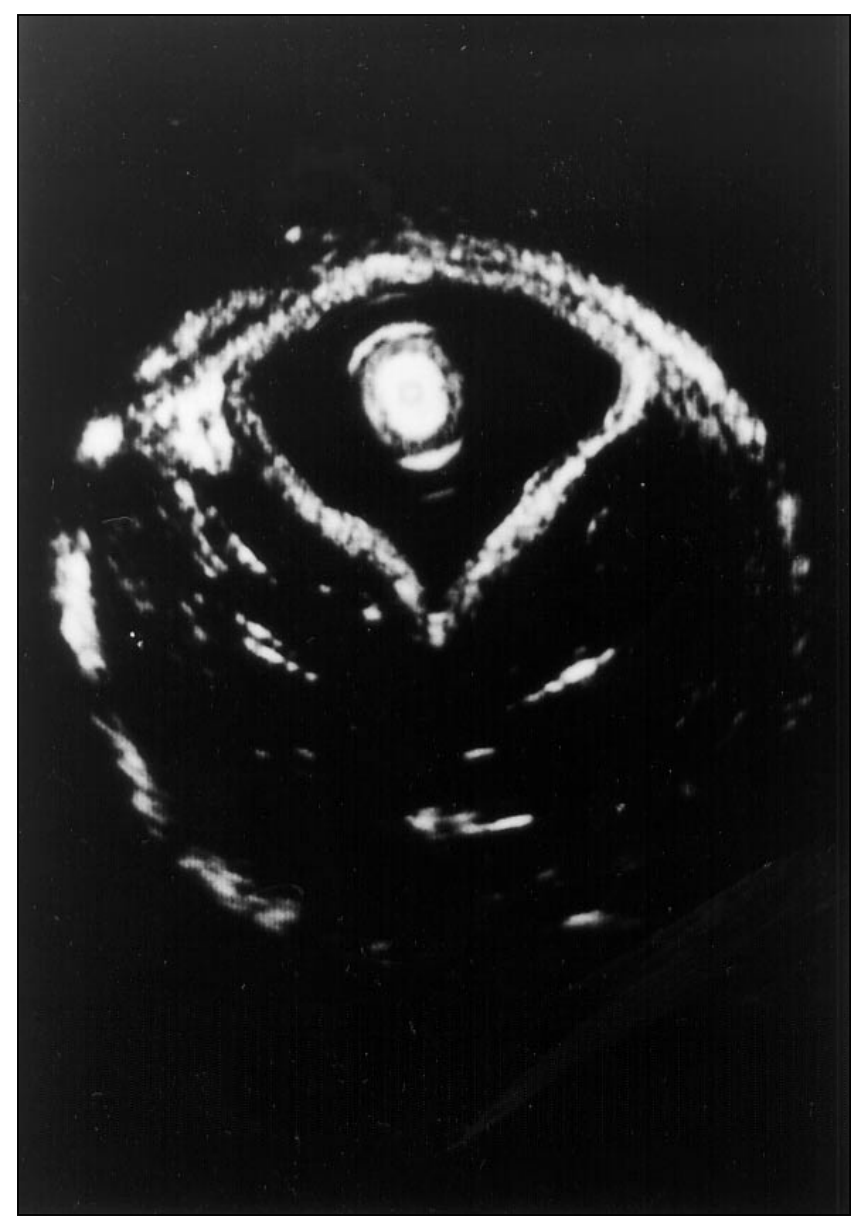

Figure 3) Ultrasonographic picture of submucosal saline injection (cross-sectional view of the esophagus). The $20 \mathrm{~mL}$ volume of saline that is submucosally injected causes more than half-circumferential lifting of the mucosa

sidered to be technically simple and can be applied to lesions anywhere in the gastrointestinal tract $(8-10)$. Other techniques of mucosal resection have also been advocated (11-13).

\section{EMRC TECHNIQUE}

In EMRC, a cap is attached to the tip of a forward-view endoscope and is fixed tightly by an adhesive tape. This cap is commercially available in Europe and Asia (pending approval in the United States, Canada and Latin America). Electrocautery should be used on the mucosa to place markings that surround the margin of the lesion. This process is not necessary in the colon.

Adrenaline saline solution diluted 500,000 times is then submucosally injected. The total volume of injected saline depends on the lesion size. It is necessary to inject at least enough saline to lift the whole lesion (Figure 1, top).

Prelooping the snare wire along the rim of EMRC cap is the next step (Figure 1, middle); a small diameter snare is essential for smoothness. We use a specially devised snare wire SD-7P from Olympus (Tokyo, Japan). The normal mucosa is treated with moderate suction to seal the outlet of the cap. Then the snare wire, which passes through the instrumental 
channel of the endoscope, is opened. The opened snare wire is fixed along the rim of the cap, with the outer sheath of the snare sticking out beyond the rim.

The target mucosa, including the lesion, is then fully sucked inside the cap and strangulated by the snare wire. At this point the strangulated mucosa looks like a polypoid lesion (Figure 1, middle). The pseudopolyp of strangulated mucosa is cut by electrocautery (Figure 1, bottom). The resected specimen can be easily removed by keeping it inside the cap without using grasping forceps.

If additional resection is necessary, the whole procedure should be repeated.

\section{RESULTS}

More than 142 patients with early stage cancer were treated by mucosal resection, mainly EMRC. Seventy-two per cent of all cases had an absolute indication for mucosal resection according to our criteria. The remainder were relatively indicated cases of mucosal resection because of poor risk of surgery or refusal of surgery. In absolutely indicated cases no local or distant metastasis was identified during the followup period. Five-year survival rate was $95 \%$; all who died suffered from other diseases such as myocardial infarction, liver cirrhosis and apoplexy.

One patient in our early series was perforated during the second cauterization without additional submucosal saline injection. With conservative treatment, such as intravenous hyperalimentation and antibiotic administration, this patient recovered and has no concomitant problems. Seven years later, she is healthy with no accident-related complications.

One patient who received near total circumferential mucosal resection developed persistent stenosis that could not be resolved by repeated forceful balloon dilation; this patient was finally treated by surgical esophagectomy.

In patients with stomach cancer, more than 102 cases received the same procedure; we encountered no major complications. In three cases who had the lesion on the lesser curvature of the gastric body, the resected specimen included small particles of muscle component. In all other save two cases the lesions were successfully treated by the initial EMR. Two cases had residual lesions after EMR and were successfully treated by laser ablation therapy. During a follow-up period of more than five years, we encountered no local recurrence of tumour in absolutely indicated cases of EMR.

\section{COMMENTS}

Mucosal cancer in the gastrointestinal tract was first diagnosed in gastric cancer, mainly by Japanese researchers. $\mathrm{Mu}$ cosal cancer in the stomach has an extremely good long term prognosis after treatment, and much effort to detect early disease in colon and esophagus has been applied. Tada and coworkers (9) advocated EMR as a local but curative treatment. We had already used laser ablation and radiation therapy as local treatments, but EMR has the advantage of acquiring specimens for histological diagnosis. The technique of Tada et al is now a standard EMR procedure, with the combination of submucosal saline injection and snare strangulation. In order to widen the application of EMR and make the procedure technically easier, we have developed the EMRC procedure.

In our experiences with the EMRC procedure we can resect any lesion from pharynx to rectum; no special additional skills are required for a standard endoscopist - the transparent cap creates enough space in front of the endoscopic lens, guaranteeing unobstructed sight even in the torturous portion of the gastrointestinal tract $(9,10)$. The principle of this procedure of 'suction and strangulation' is based on the endoscopic variceal ligation procedure developed by Stiegmann et al (14).

One of the most serious complications of EMRC is perforation. As indicated in Figure 2, whole layers, including the muscle layer, are completely sucked inside the cap when there is no submucosal saline injection. The most risky factor that may causes perforation is, we suspect, lack of volume of submucosal saline injection (Figure 2). In the esophagus, approximately $20 \mathrm{~mL}$ of saline causes more than half-circumferential mucosal dissection from the muscle layer, which makes mucosal resection safer (Figure 3 ). In the stomach the same phenomenon is experienced. It is possible that if saline is accurately injected into the submucosa, lifting of mucosa or bulging of mucosa can always be observed in any part of the gastrointestinal tract.

The other major complication of EMRC is persistent stenosis after healing of an artificial ulcer. This serious problem can occur when near total circumferential resection is preformed in the esophagus and prepylorus; therefore, one should avoid near total or total mucosal resection.

In almost all cases of mucosal resection, the patient's quality of life can be maintained (15), so we believe that early detection of cancerous lesion and EMR treatment are ideal goals of cancer treatment.

\section{REFERENCES}

1. Endo M, Ide H, eds. Endoscopic Staining In Early Diagnosis of Esophageal Cancer, 1st edn. Tokyo: Japan Scientific Societies Press, 1991:1-70.

2. Endo M, Kawano T. Analysis of 1125 cases of early esophageal carcinoma in Japan. Dig Endosc 1991;4:71-6.

3. Nakajima T. [Tabular analysis of 10,000 cases of gastric cancer in CIH.] Jpn J Cancer Chemother 1994;21:1813-97.

4. Rosenberg N. Submucosal saline wheal as safety factor in fulguration of rectal and sigmoidal polypi. Arch Surg 1955;70:120-2.

5. Deyhle P, Largiader F, Jenny S, Fumagalli I. A method of

endoscopic electroresection of sessile colonic polyps. Endoscopy 1973;5:38-40.

6. Takemoto T, Tada M, Yanai H, Karita M, Okita K. Significance of strip biopsy with particular references to endoscopic "mucosectomy". Digest Endosc 1989;1:4-9.

7. Inoue H, Endo M. Endoscopic esophageal mucosal resection using a transparent tube. Surg Endosc 1990;4:198-201.

8. Inoue H, Takeshita K, Hori H, Muraoka Y, Yoneshima H, Endo M. Endoscopic mucosal resection with a cap-fitted panendoscope for esophagus, stomach and colon mucosal lesions. Gastrointest Endosc 1993;39:58-62. 
9. Tada M, Inoue H, Yabata E, Okabe E, Endo M. Colonic mucosal resection using a transparent cap-fitted endoscope. Gastrointest Endosc 1996;44:63-5.

10. Izumi Y, Teramoto K, Ohshima M, et al. Endoscopic resection of duodenal ampulla with a transparent plastic cap. Surgery 1998;123:109-10.

11. Monma K, Sakaki N, Yoshida M. [Endoscopic mucosectomy for precise evaluation and treatment of esophageal intraepithelial cancer.] Endoscopia Digestiva 1990;2:501-6.

12. Makuuchi H, Machimura T, Sugihara T, et al. [Endoscopic diagnosis and treatment of mucosal cancer of the esophagus.] Endoscopia Digestiva 1990;2:447-52

13. Kawano T, Miyake S, Yasuno M, et al. A new technique for endoscopic esophageal mucosectomy using a transparent overtube with intraluminal negative pressure. Digest Endosc 1991;3:159-67.

14. Stiegmann GV, Cambre T, Sun JH. A new endoscopic elastic band ligating device. Gastrointest Endosc 1986;32:230-3.

15. Takeshita K, Tani M, Inoue H, et al. Endoscopic treatment of early oesophageal or gastric cancer. Gut 1997;40:123-7. 


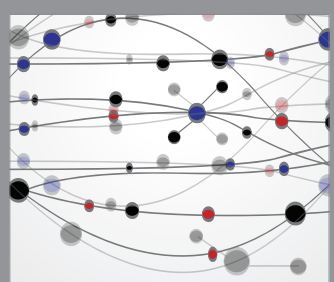

The Scientific World Journal
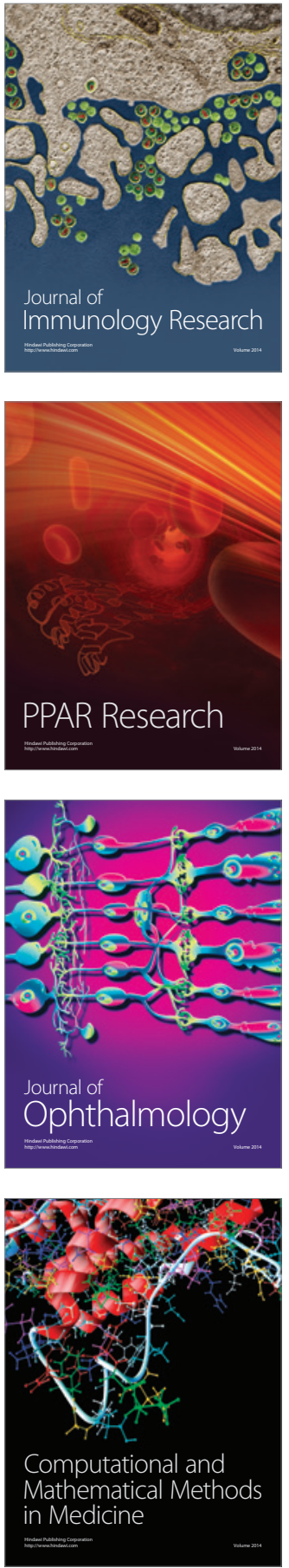

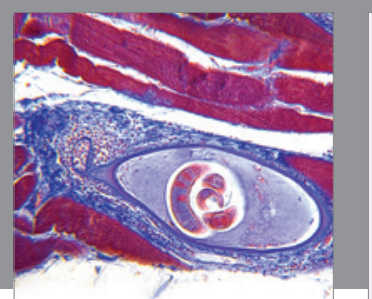

Gastroenterology Research and Practice

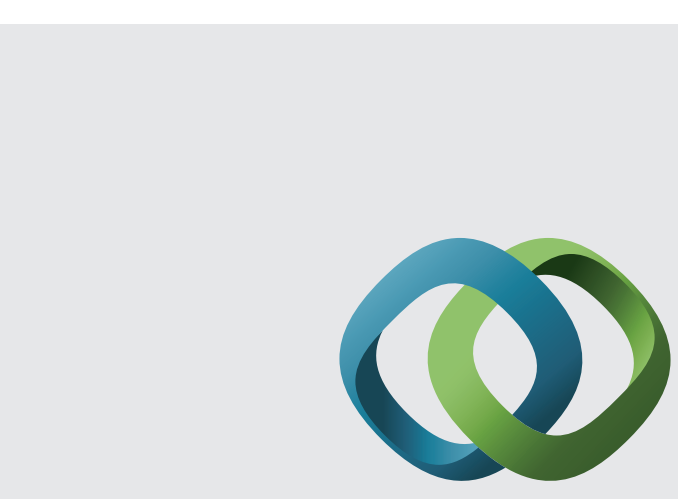

\section{Hindawi}

Submit your manuscripts at

http://www.hindawi.com
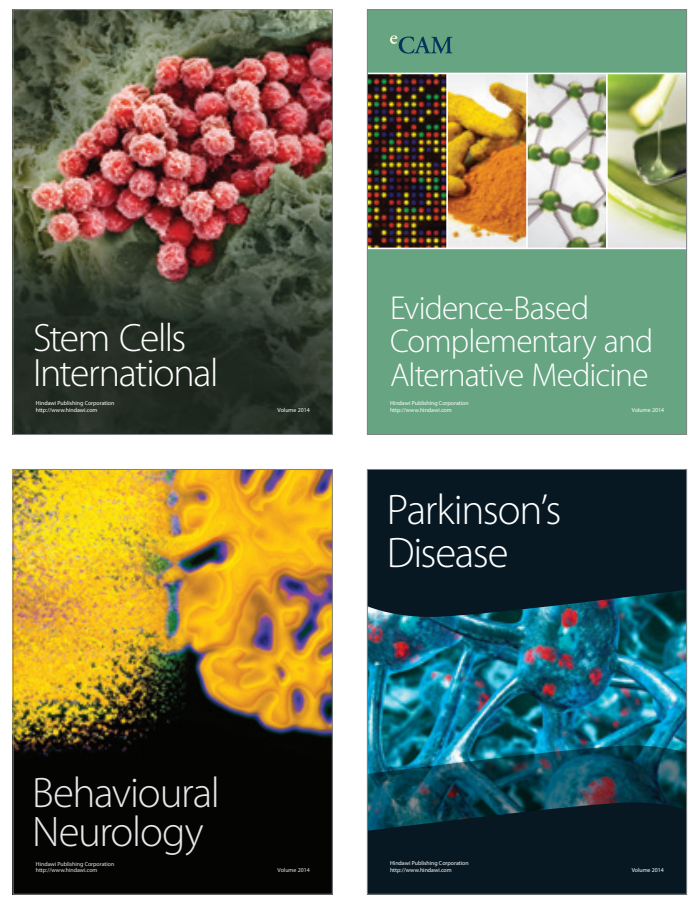
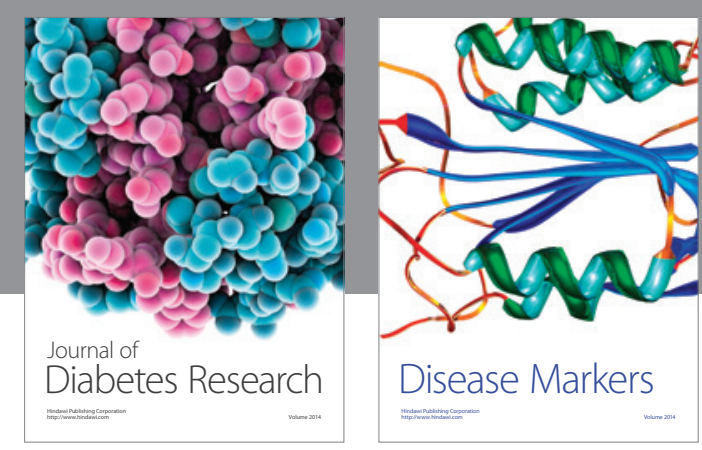

Disease Markers
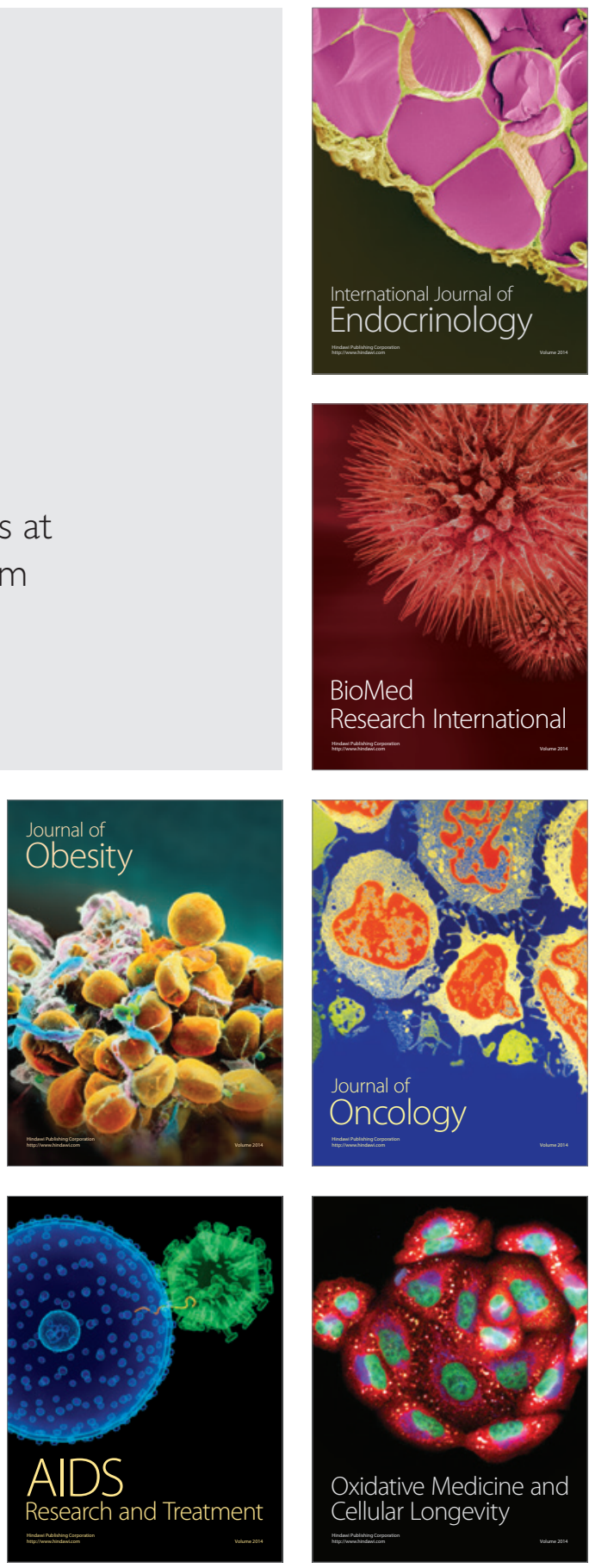